Reprod. Nutr. Dévelop., 1982, 22 (5), 713-734.

\title{
La gynogenèse chez les vertébrés
}

\section{CHOURROUT}

Laboratoire de Physiologie des Poissons, I.N.R.A., 78350 Jouy-en-Josas, France.

Summary. Gynogenesis in vertebrates.

After a rapid description of five gynogenetic unisexual species, this paper reviews the methods used for inducing gynogenesis in bisexual species.

1) The frequency of male genome extrusion, very low after intraspecific mating, can be appreciably increased in some particular interspecific combinations ; cold-shocking the eggs at the time of fertilization can also result in gynogenetic development. In the case, of mammals, the male pronucleus can be microsurgically removed, but in most cases (amphibians, fish), haploid gynogenesis is induced by mutagenic treatment of the sperm (gamma, X, UV irradiation; chemicals) before fertilization.

2) Viable gynogenesis depends on the possibility of diploidizing the female genetic set ; several factors (oocyte aging, genetic factors, induction of ovulation) may be responsible for the high frequency of "spontaneous " diploidization reported in some studies. But diploid gynogenesis is generally obtained by induced retention of the second polar body or by induced suppression of the first cleavage, achieved by heat or pressure treatment of eggs (fish and amphibins) or by the use of antimitotic chemicals (mammals).

Diploid gynegenesis results in high inbreeding levels and original sex ratios. In the discussion, we have tried to show that the objectives which stimulated its induction in amphibians have been frequently unattained or reached in other ways. In commercial fish species, gynogenesis may in the future contribute to the solution of major problems such as genetic improvement and control of reproduction.

\section{A. Introduction}

La gynogenèse est le développement d'un œuf activé par un spermatozoïde, mais sans participation du génome paternel. Sur le plan génétique, elle est équivalente à la pathénogenèse, mais celle-ci est déclenchée par d'autres facteurs.

La gynogenèse est le mode de reproduction de plusieurs vertébrés inférieurs, et si l'on attribuait autrefois à ce phénomène une valeur relativement anecdotique, on peut lui accorder aujourd'hui une réelle importance en tant que mécanisme évolutif.

Depuis le début du siècle, un grand nombre d'auteurs ont montré que la gynogenèse pouvait être induite chez de nombreuses espèces d'amphibiens ; si 
les techniques utilisées se sont sensiblement améliorées et diversifiées, la méthode générale reste la même : elle consiste à induire un développement haploïde de l'œuf, généralement à la suite d'un traitement mutagène des spermatozoïdes utilisés pour l'activation ; dans un deuxième temps, un traitement approprié des œufs inséminés compense l'absence du pronucléus mâle, en doublant le matériel génétique de l'embryon le plus souvent lors de la seconde division de méiose, et plus rarement lors de la première mitose.

Ce mode de reproduction assure une augmentation rapide du niveau de consanguinité, d'où sa première utilisation chez les amphibiens pour mettre en évidence des mutations récessives, permettant d'élucider certains mécanismes physiologiques, mais également fournissant des souches d'animaux facilement reconnaissables. La gynogenèse, par le même fait est une voie a priori efficace de création de lignées homogènes et histocompatibles, précieuses pour divers types d'expérimentation.

D'autre part, elle fournit des descendances de sex-ratio originales, caractéristiques du mode de déterminisme du sexe de l'espèce étudiée : dans le cas d'une homogamétie femelle, elle conduit à des descendances uni sexuées (sexe génétique) ; dans le cas d'une homogamétie mâle, elle fournit un génotype sexuel nouveau (WW) viable chez les vertébrés inférieurs.

Signalons enfin, que dans le cas d'une diploïdisation par rétention du second globule polaire, le génome obtenu équivaut à la fusion de deux produits terminaux de méiose identiques aux post-réductions près : il est donc possible grâce à la gynogenèse d'estimer le taux de recombinaisons survenues entre certains gènes et leur centromère, et d'apporter par conséquent une contribution tout à fait originale, chez les organismes diploïdes, à la cartographie génétique.

Depuis les années soixante, plusieurs équipes ont entrepris la transposition de ces techniques chez plusieurs espèces de poissons d'intérêt économique. Généralement, ces études avaient pour but la production rapide de lignées consanguines dont le recroisement peut aboutir, comme chez certaines plantes allogames, à d'importants effets d'hétérosis : l'application de croisements frère-sœur successifs apparaît en effet ici très laborieuse, compte tenu de l'intervalle de génération important ( 2 ans ou plus) des espèces considérées. D'autre part, dans la mesure où les performances du sexe femelle sont les plus intéressantes chez les salmonidés (maturation plus tardive) et chez les cyprinidés (croissance plus rapide) dans certaines situations d'élevage, et dans la mesure où aucun gène à action visible permettant un sexage précoce n'a été détecté jusqu'aujourd'hui, la gynogenèse paraît pouvoir s'insérer efficacement dans des schémas de production de cheptels unisexués.

Nous examinerons rapidement le cas des espèces gynogénétiques naturelles puis l'ensemble des méthodes ayant aboutit à l'induction de la gynogenèse chez les amphibiens, les mammifères et les poissons.

Enfin, nous tenterons de montrer que si les perspectives offertes au laboratoire par la gynogenèse induite ont toujours été très limitées, son champ d'application paraît plus large chez les poissons, tout au moins dans le cadre de l'amélioration génétique. 


\section{B. La gynogenèse naturelle}

II s'agit d'espèces unisexués femelles se reproduisant de manière systématique par gynogenèse. Elles appartiennent aux groupes des amphibiens et des poissons, la parthénogenèse étant réservée aux seuls amniotes. Nous décrirons brièvement un exemple pris chez les amphibiens et quatre exemples pris chez les poissons, puis nous tenterons d'en dégager quelques généralités.

1. Le cas des urodèles gynogénétiques (Uzzell, 1964, 1970).

Ambystoma platineum et $A$. tremblayi sont des espèces triploïdes vraisemblablement nées d'une hybridation entre deux espèces diplö̈des : A. laterale et $A$. jeffersonium $(A$. platineum possède deux stocks génétiques de $A$. jeffersonium; $A$. tremblayi possède deux stocks génétiques de $A$. laterale). Dans les deux cas, une endomitose préméiotique permet la production d'œufs triploïdes.

2. Le cas de Poeciliopsis Cy.

II s'agit à nouveau de deux espèces triploüdes, issues du croisement de $P$. monacha et de $P$. lucida. Ici encore, c'est une endomitose préméiotique (Cimino, 1972) qui permet de compenser la réduction chromatique. Les œufs triploïdes sont activés par des spermatozoïdes provenant de l'une de espèces parentales. On peut noter qu'il existe une espèce unisexuée femelle diploïde appartenant au même genre, et qui se reproduit par hybridogenèse (Schultz, 1967).

3. Le cas de Poecilia formosa.

La situation est ici plus complexe. Poecilia formosa est une espèce hybride entre $P$. latipinna et $P$. mexicana (Hubbs et Hubbs, 1932 ; Hubbs, 1961) composée de populations diploïdes et triplö̈des; à l'intérieur de chaque population, les individus sont histocompatibles (Kallman, 1962). Le mécanisme compensateur de la réduction méiotique n'a pas été élucidé. Des individus triploïdes apparaissent parfois dans la descendance de populations diploïdes, traduisant ainsi le taux d'erreur du processus de gynogenèse (Rasch et al., 1965; Schultz et Kallman, 1968).

4. Le cas des carassins gynogénétiques.

Carassius auratus gibelio est une espèce originaire d'Europe centrale composée de populations diploïdes (Lieder, 1959) et triploïdes (Cherfas, 1965 a) Carassius auratus langsdorfii est une espèce japonaise composée de populations triploïdes et peut-être tétraploïdes (Kobayasi, 1976). Dans les deux cas, l'activation est sans doute réalisée par des mâles de cyprinidés sympatriques. Contrairement aux exemples précédents, il n'y a pas d'indication, ni d'études démontrant la structure hybride de leurs génomes. Enfin, malgré les propositions faites pour rendre compte de la production d'ovules triploïdes par Cherfas (1965 a), qui décrit la formation d'un fuseau méiotique tripolaire chez C. a. gibelio, et Kobayasi (1976), à savoir l'existence d'une seule division méiotique chez $C$. a. langsdorfii, cette question ne semble pas vraiment résolue.

\section{Discussion.}

Un certain nombre de points se dégagent de l'étude de ces différents cas : 1. - Le génome des espèces gynogénétiques naturelles apparaît résulter d'une 
hybridation ancienne entre deux espèces voisines. Si, nous supposons que, au moins dans une zone donnée, cette hybridation avait lieu à un taux important, tout mécanisme permettant la reproduction conforme de ce génome hybride apparaissait doté d'une valeur sélective élevée, puisqu'il évitait la production de gamètes déquilibrés.

2. - La stimulation gynogénétique des ovules est provoquée par les spermatozoïdes d'une autre espèce.

3. - Un mécanisme cytologique original permet de conserver la ploïdie : lorsqu'il s'agit d'une endomitose préméiotique, il conduit à une multiplication conforme et donc à des populations formées de clones à hétérozygotie fixée.

Deux phénomènes apparaissent fréquemment associés à la gynogenèse naturelle :

1. - L'hybridogenèse, qui permet également la conservation d'une génome hybride par le biais d'un méiose sélective.

2. - La triploïdisation, qui résulte du caractère non absolu de l'exclusion du génome mâle. Notons que ce phénomène peut être récurrent et conduire à une évolution par polyploïdisations successives (cas des carassins).

Enfin, notons que le caractère unisexué d'une population gynogénétique ne constitue pas un argument en faveur d'une homogamétie femelle : en effet, dans le cas d'une endmitose préméiotique avec appariement de chromosomes dupliqués, la conservation d'une formule de type $Z_{1} W_{2}$ (avec $Z_{1}$ issu de l'espèce $E_{1}$, et $W_{2}$ issu de l'espèce $E_{2}$ ) apparaît tout à fait possible.

\section{La méthode générale d'induction}

Elle consiste à induire un développement haplö̈de (individu non viable chez les vertébrés), généralement à la suite du traitement des spermatozoïdes utilisés pour l'activation. Un traitement approprié des œufs fécondés compense ensuite l'absence du pronucleus mâle, en doublant le matériel génétique de l'embryon, soit lors de la seconde division de méiose, soit lors d'une des premières mitoses de segmentation. Un certain nombre de critères rendent la mise au point de ces deux traitements plus aisée chez certaines espèces :

- La maîtrise de la fécondation in vitro (contrôle de l'émission des gamètes, bon taux de fécondation et de survie embryonnaire).

- Une fertilité femelle élevée.

- Un développement embryonnaire rapide et de chronologie bien connue, surtout dans ses premiers stades.

- Une identification facile des produits au niveau caryologique (techniques existantes, petit nombre de chromosomes, paires d'homologues reconnaissables), et au niveau du nombre de gènes identifiables (gènes à action visible, systèmes biochimiques).

\section{L'exclusion du génome mâle}

1. La gynogenèse spontanée dans un croisement intraspécifique.

D'après Fankhauser et Humphrey (1959), I'haploïdie spontanée serait l'un des 
accidents de développement les plus courants chez les amphibiens 10,42 p. 100 sur 109445 œufs chez l'axolotll ; l'utilisation de mâles et de femelles de colorations différentes permet de préciser que les embryons considérés sont en majorité d'origine gynogénétique, et en minorité d'origine androgénétique (Humphrey et Fankhauser, 1957).

Austin et Braden (1954) ont identifié chez le rat deux œufs haploïdes (stades 1 cellule et 2 cellules) dont l'origine gynogénétique semble indiscutable (présence dans l'œuf d'un acrosome, d'une pièce intermédiaire et d'un pronucleus mâle dégénérescent).

Il est évident, que le terme spontané qualifie un certain nombre d'anomalies non provoquées intentionnellement, mais probablement issues d'un ensemble de conditions très particulières (qualité des gamètes notamment) qu'il serait très intéressant d'identifier.

\section{La gynogenèse spontanée dans un croisement interspécifique.}

Des individus gynogénétiques ont souvent été recensés à la suite d'inséminations interspécifiques ; leur détection est d'autant plus aisée que l'hybride est précocément inviable. Ainsi, Stanley (1976) obtient à partir du croisement « femelle carpe commune $\times$ mâle carpe chinoise " effectué sur 100000 œufs) quelques survivants à l'âge de trois mois, dont cinq individus de phénotype carpe commune. Buss et Wright (1956) recensent uniquement des survivants d'apparence saumon de fontaine à la suite du croisement " femelle saumon de fontaine $\times$ mâle truite fario "; mais là encore, leur fréquence est très faible et leur identification n'est pas certaine. Enfin, Ferrier (1966) montre que les rares survivants du croisement « femelle Pleurodeles waltlii $\times$ mâle Salamandra salamandra " sont gynogénétiques et de ploïdies variables $(2 n, 3 n, 4 n, 5 n)$. Pour ces trois exemples les haploïdes n'étant pas plus viables que les hybrides, seuls sont détectés des individus gynogénétiques diploïdes ou polyploïdes, et le taux d'exclusion du génome mâle est donc largement sous-estimé ; en revanche, il n'est pas démontré qu'il soit plus élevé que dans le cas d'une combinaison intraspécifique.

II semble cependant que certains croisements s'accompagnent d'une gynogenèse sinon systématique, toutefois très fréquente : tous les embryons de l'hybridation "femelle Rana pipiens × mâle Scaphiopus holdbrooki " sont démontrés haploïdes gynogénétiques par examen caryologique (Ting, 1951 ; Volpe et Dasgupta, 1962). Purdom et Lincoln (1974) assurent qu'il en est de même pour le croisement "femelle plie $\times$ mâle flétan » qui donne une descendance anormale qu'ils supposent entièrement composée d'haplö̈des. Il nous semble cependant que l'hypothèse selon laquelle une partie de ces embryons seraient des hybrides ne puisse être rejetée : Purdom et Lincoln, pour appuyer leur certitude, soumettent les œufs peu après la fécondation à un choc froid prolongé pour induire la rétention du second globule polaire, et ils obtiennent ainsi un bon nombre de survivants de phénotype plie ; nous verrons par la suite que de tels traitements favorisent entre autres l'exclusion du pronucleus mâle et sont peut-être dans ce cas à l'origine de la gynogenèse.

Enfin, signalons pour les mammifères le cas d'un embryon haploïde (6 cellules) obtenu après injection de sperme de taureau dans les trompes d'une lapine (Pincus, 1939). 
3. Facteurs génétiques d'exclusion du pronucleus mâle.

Dans les exemples précédents, il est probable qu'une attaque enzymatique ou un rejet du pronucleus mâle, ou encore que certaines particularités anatomiques ou physiologiques des gamètes combinés sont à l'origine de la gynogenèse.

Des embryons ont été obtenus en nombre dans les descendances de lignées de souris " silver " fournissant un taux élevé d'hétéroploïdie (Beatty et Fischberg, 1951 ; Fischberg et Beatty, 1952 a). D'autre part, le fait qu'on utilise une mutation provoquant l'exclusion du pronucleus mâle pour obtenir à volonté la gynogenèse chez la drosophile, illustre également bien le fait que l'on pourrait envisager l'exploitation de telles particularités génétiques, par exemple par mutagenèse ou par sélection, dans quelques cas très particuliers.

\section{L'induction de la gynogenèse par choc froid.}

Rostand $(1934,1936)$ a constaté que certaines hybridations entre anoures (" femelle Bufo vulgaris $\times$ mâle Rana temporaria "; " femelle Hyla arborea $\times$ mâle Rana temporaria ") ordinairement létales avant le stade gastrula, donnent des larves lorsque les œufs fécondés ont été soumis à un refroidissement prolongé ; quelques-unes de ces larves dépassent la métamorphose et sont supposées diploïdes. Bien que ces expériences ne soient pas présentées dans le détail, il semble que les larves soient gynogénétiques, le choc froid provoquant à la fois la rétention du second globule polaire et l'exclusion du pronucleus mâle.

Humphrey et Fankhauser (1957) ont montré qu'à la suite d'un choc froid de durée supérieure à neuf heures, les œufs de Triturus pyrrhogaster donnent naissance à une majorité d'embryons haploïdes (63 sur 110), mais dont l'origine est androgénétique.

\section{Le cas particulier des agents initiateurs de clivage.}

Bataillon (1911) a obtenu des développements embryonnaires avancés en piquant des œufs de Bufo vulgaris avec une aiguille souillée par le contact de divers tissus d'animaux (sang de grenouille, de carpe et de rat ; sperme de carpe, etc...), alors que le traumatisme occasionné par une aiguille propre ne déclenche jamais, à lui seul, de clivage régulier.

Plusieurs auteurs ont récemment extrait de tissus très divers des fractions microtubulaires capables d'initier un développement régulier chez Rana pipiens (Fraser, 1971) et chez Oryzias latipes (Iwamatsu et Ohta, 1974); dans une certaine mesure, on peut considérer les développements obtenus comme intermédiaires entre gynogenèse et parthénogenèse, dans le cas où les extraits proviennent de spermatozoïdes.

Cette technique ne semble pas immédiatement utilisable pour produire massivement des développements haploödes ; elle est particulièrement délicate à mettre en œuvre chez les poissons, où l'injection passe nécessairement par le micropyle.

\section{L'exclusion du pronucleus mâle par microchirurgie.}

Le prélèvement du noyau ovocytaire a souvent été effectué comme préalable à la greffe nucléaire chez les amphibiens. Cette technique a été récemment transposé par Modlinski (1975) sur l'œuf fécondé de souris, duquel il extrait l'un des pronuclei (une fois sur deux le pronucleus mâle) peu de temps avant la caryogamie. 
Outre le fait que cette opération constitue un tour de force technique, le pourcentage d'œufs lésés par la micropipette est très élevé, et les embryons obtenus sont aussi bien androgénétiques que gynogénétiques.

\section{Le traitement du sperme par les rayons ionisants.}

Les traitements mutagènes du sperme sont les plus fréquemment utilisés pour produire des développements gynogénétiques haploïdes; les rayons ionisants notamment, très pénétrants, assurent l'inactivation homogène de volumes importants de sperme pur.

Hertwig, en 1911, en soumettant du sperme de Rana fusca à l'action d'une source de radium, mit en évidence un effet dose sur la mortalité embryonnaire qu'il qualifia de paradoxal et qui porte aujourd'hui son nom : pour de faibles doses croissantes, la mortalité atteint progressivement 100 p. 100, puis diminue pour les fortes doses. Oppermann, en 1913, en reprenant la même méthode chez la truite fario, obtint les mêmes résultats : il reconnut chez les embryons correspondants aux longues durées d'irradiation des anomalies analogues à celles décrites par Bataillon sur les grenouilles parthénogénétiques et montra que la taille de leurs noyaux somatiques était plus faible que cele de noyaux d'embryons témoins ; il est par conséquent abusif de présenter ces auteurs comme ignorants du processus de gynogenèse qu'ils avaient induit.

II semble que ce soit Rugh, en 1939, qui reprit le premier ces expériences en inactivant le sperme de Rana pipiens à l'aide d'une source de rayons $\mathrm{X}$. Il obtint les mêmes résultats avec Exner (1940), mais en utilisant sur les œufs de Rana pipiens le sperme irradié d'une espèce voisine (sperme hétérologue). Briggs et al. (1951) en irradiant du sperme de Rana pipiens et de Rana catesbeiana à des doses comprises entre 65000 et 100000 rads, provoquèrent des développements gynogénétiques haploïdes de Rana pipiens : ils montrèrent que la survie des embryons provenant de sperme homologue ou hétérologue était la même ; et que dans certains cas, le pronucleus mâle endommagé pouvait se placer au centre du fuseau de la première mitose et se retrouver sous forme de fragments répliqués dans les cellules de l'embryon.

Aujourd'hui, les rayons ionisants sont de moins en moins utilisés chez les amphibiens, du fait des problèmes de coût et de sécurité que posent la maintenance d'une source radioactive.

Ces résultats contrastent avec ceux d'expériences plus récentes conduites chez les mammifères : ni Edwards (1956 a) chez la souris avec les rayons $X$, ni Chang et al. (1957) chez le lapin avec les rayons gamma, ni Simon (1972) chez le lapin avec les rayons $X$, n'ont pu obtenir de gynogenèse haploïde de manière incontestable. Il semble qu'aussi bien in vitro qu'in vivo, le pouvoir fécondant du sperme de mammifères ne puisse résister aux doses inactivantes.

En revanche, les rayons ionisants sont utilisés couramment pour induire la gynogenèse chez les poissons. Lasher et Rugh (1962) ont décrit l'effet Hertwig chez Fundulus heteroclitus d'une manière plutôt qualitative (description des anomalies de développement). Romashov et al. (1961), Purdom (1969), Nagy et al. (1978) préconisent l'utilisation de 100000 rads de rayons $X$ ou gamma pour inactiver le sperme de plusieurs espèces d'élevage. Nous avons de notre côté optimisé 
le traitement gamma chez la truite arc-en-ciel (Chourrout et al., 1980) et avons retenu des doses plus élevées.

8. Le traitement du sperme par les rayons ultraviolets.

Dalcq et Simon (1931) sur Rana fusca, ont les premiers décrit les variations du taux de mortalité embryonnaire consécutives à l'irradiation par les ultraviolets du sperme à des doses variables. Pincus et Enzmann (1936) n'ont pas obtenu de développements gynogénétiques par cette méthode chez le lapin ; par contre, il semble qu'Edwards (1956 b) ait produit des haploïdes chez la souris, mais toujours en minorité par rapport aux embryons non gynogénétiques (diploïdes et hypodiploïdes) issus du même échantillon de sperme.

Le traitement par les rayons ultraviolets du sperme est aujourd'hui couramment utilisé chez les amphibiens et de plus en plus chez les poissons (tabl. 1) ; il est réalisé à l'aide de lampes germicides ordinaires.

Pogany (1976) a étudié chez Rana pipiens l'effet dose et obtenu un « pseudoeffet Hertwig " : il n'a pas mis en évidence de doses moyennes conduisant comme dans le cas des rayons ionisants, à une mortalité embryonnaire totale ; le faible pouvoir pénétrant du rayonnement semble responsable de la persistance d'embryons diploïdes (minoritaires toutefois) pour les doses les plus élevées ; des embryons hypodiploïdes sont également recensés pour certaines durées d'irradiation.

Un certain nombre d'artifices permettent d'augmenter I'homogénéité du traitement : utilisation de faibles épaisseurs de sperme, dilution, et agitation de l'échantillon pendant l'irradiation; dans de nombreux cas, ces modalités ne sont

TABLEAU 1

Références récentes de travaux concernant le traitement du sperme par les rayons ultraviolets

a. Sperme homologue

\begin{tabular}{|c|c|c|c|}
\hline Auteurs & Espèce & Marqeur mendelien & Det. niveau de ploïdie \\
\hline Jaylet (1972) & P. waltiii & oui & caryotype \\
\hline Farina (1976) & D. pictus & oui & non \\
\hline Pogany (1976) & R. pipiens & non & caryotype \\
\hline Nace et al. (1970) & R. pipiens & oui & non \\
\hline Trottier et Armstrong (1976) & $x$. laevis & oui & nb. nucléoles \\
\hline Kawahara (1978) & X. laevis & non & non \\
\hline Tompkins (1978) & X. laevis & oui & nb. nucléoles \\
\hline Reindschmidt et al. (1979) & X. laevis & oui & $?$ \\
\hline Streisinger et al. (1981) & B. rerio & oui & $?$ \\
\hline Chourrout (non publié) & S. gairdneri & oui & caryotype \\
\hline
\end{tabular}

\section{b. Sperme hétérologue}

\begin{tabular}{llll}
$\begin{array}{l}\text { Nace et al. (1970) } \\
\text { Jaylet (1972) }\end{array}$ & $\begin{array}{l}\text { R. pipiens } \\
\text { P. waltii }\end{array}$ & $\begin{array}{l}\text { R. clamitans } \\
\text { S. salamandra }\end{array}$ & $\begin{array}{l}\text { non } \\
\text { caryotype }\end{array}$ \\
\hline $\begin{array}{lll}\text { Staniey et Jones (1976) } \\
\text { Chourrout (non publié) }\end{array}$ & $\begin{array}{l}\text { C. idella } \\
\text { E. lucius }\end{array}$ & $\begin{array}{l}\text { C. carpio } \\
\text { S. gairdneri }\end{array}$ & $\begin{array}{l}\text { non } \\
\text { non }\end{array}$ \\
\hline
\end{tabular}


pas décrites dans le détail, et leur multiplication rend difficile la répétition de l'expérience dans d'autres laboratoires. De plus ni le dénombrement des nucléoles de cellules embryonnaires, ni l'utilisation d'un marqueur mendélien, ne sont des preuves irréfutables de l'exclusion totale du génome mâle, c'est-à-dire de l'haploïdie stricte.

Une méthode intéressante consiste à utiliser un sperme hérérologue qui conduit sans irradiation à un hybride précocément abortif ; dans ce cas, les auteurs qui ont soumis les œufs fécondés par du sperme irradié à un traitement de rétention du second globule polaire, concluent que tout individu dépassant la métamorphose est gynogénétique diploïde ; pourtant, il semble nécessaire d'identifier ces produits par examen caryologique, pour confirmer l'absence de résidus de chromatine mâle. Notons enfin que cette stratégie devient caduque, s'il est démontré que l'hybride triploïde est viable (contrairement à l'hybride diploïde).

Pour d'autres raisons l'utilisation de sperme hérérologue peut être préférée à celle de sperme homologue : Jaylet (1972) a montré que la chromatine mâle qui persiste sous forme de menus fragments chromosomiques dans les cellules de certains embryons obtenus avec du sperme homologue irradié aux rayons $X$ est exclue si les œufs de Pleurodeles waltlii sont fécondés par du sperme irradié de Salamandra salamandra.

Globalement, nous voyons donc que si l'emploi d'une lampe à rayons ultraviolets est plus facile que celui d'une source radioactive, il nécessite certaines précautions supplémentaires, en raison du faible pouvoir pénétrant des rayons qu'elle produit.

\section{Le traitement du sperme par un mutagène chimique.}

Hertwig (1924) semble être le premier auteur à avoir traité du sperme de grenouille avec la trypaflavine, un mutagène à action photodynamique. La réussite des expériences conduites postérieurement chez les amphibiens (Dalcq, 1931 et Drebinger, 1951 avec la trypaflavine; Briggs et al., 1951 avec le bleu de toluidine; Drebinger, 1951 avec la moutarde à l'azote) contraste avec les mauvais résultats enregistrés chez les mammifères (Thibault et Ortavant, 1949 ; Edwards, 1958).

De récents travaux ont mis en évidence un effet Hertwig consécutif au traitement du sperme de poissons par deux mutagènes alkylants (Tsoy, 1969, avec la nitrosométhylurée et le diméthylsulfate).

\section{E. La diploïdie spontanée}

La plupart des auteurs ont noté à la suite de la fécondation d'un lot d'ovocytes d'amphibiens ou de poissons avec du sperme irradié qu'un nombre très faible (moins de 1 p. 100 en général) d'individus gynogénétiques dépassent la métamorphose. Leur présence a été interprétée comme le résultat d'anomalies classiques de la méiose et du développement qui conduisent à la diploïdie dont les principales sont l'endomitose préméiotique, la suppression de l'une des divisions méiotiques, ou de l'une des premières mitoses embryonnaires. Même dans le cas où ces individus ont été correctement identifiés par I'analyse caryologique et formelle, la 
fréquence des anomalies correspondantes peut difficilement être évaluée par le taux de survie à un âge avancé, puisqu'on ignore toujours les taux de mortalité spécifique qui s'exerçent sur les divers génomes haploïdes et diploïdes plus ou moins consanguins. Quoiqu'il en soit, la fréquence d'individus viables peut atteindre dans certains cas des valeurs élevées (Richards et Nace, 1977, avancent jusqu'à $35 \mathrm{p} .100$ chez Rana pipiens), et on peut sans doute relier ce phénomène aux forts taux de triploïdie parfois enregistrés à la suite d'une insémination par du sperme normal (Kawamura, 1942), encore que la dispermie puisse également rendre compte de ces dernières observations.

Nous examinerons certains facteurs susceptibles d'être la cause des déviations du taux de diploïdie spontanée en gynogenèse, puisque connus pour provoquer de fortes fréquences d'hétéroploïdies dans le cas de fécondations normales.

1. Le traitement d'induction de la superovulation.

Une baisse du taux de survie embryonnaire a souvent été enregistrée à la suite de l'induction de la superovulation chez les mammifères et plusieurs auteurs ont montré qu'elle correspond à l'induction d'un taux anormal d'hétéroploïdies. L'expérience la plus probante est celle de Maudlin et Fraser (1977), sur la souris, qui ont obtenu à la suite d'une injection de PMSG 60 embryons triploïdes et 4 embryons tétraploïdes sur 452 embryons au stade 2 cellules, contre 4 triplö̈des sur les 228 embryons témoins. De tels résultats ont été mis en évidence chez la brebis (Williams et Long, 1980). Cependant, il semble que dans les deux cas, ce soit la dispermie qui soit à l'origine de la majorité des triploïdes, et par conséquent le traitement hormonal $n$ 'aura pas a priori d'effet sur le taux de diploïdie gynogénétique spontanée.

\section{Les facteurs génétiques.}

De nombreuses mutations perturbant la disjonction méiotique ont été obtenues chez les plantes et chez la drosophile (Golubovskaya, 1979). La plupart conduisent à la stérilité ou à la production de gamètes déséquilibrés. Cependant, de forts taux de triploïdie ont été par exemple recensés dans des descendances de maïs porteur de la mutation " el ". De la même façon les lignées consanguines de souris « silver " produisent une proportion anormale d'embryons triploïdes (Beatty et Fischberg, 1951 ; Fischberg et Beatty, 1952 a, b) ; Braden (1957) a montré que ceux-ci résultaient d'une rétention du second globule polaire.

\section{Le vieillissement des ovocytes.}

On sait depuis longtemps que chez les mammifères un certain nombre d'anomalies du caryotype ont pour origine l'âge élevé de la femelle au moment de l'ovulation.

Le vieillissement des ovocytes avant la fécondation, ou fécondation retardée, est un autre facteur générateur d'hétéroploïdie. Witschi et Laguens (1963) ont montré que la plupart des embryons anormaux issus de pontes surmatures de Xenopus laevis et de Rana pipiens étaient aneuplö̈des et résultaient de perturbations de la méiose II ou des premières mitoses de la segmentation ; l'examen des embryons normaux leur aurait peut être permis de détecter un nombre important de triploïdes. En effet, Blackler et Cassidy (1980) ont examiné tous les embryons provenant de pontes de surmaturité variable (acquise in vitro) chez Xenopus laevis 
et montré l'existence d'une corrélation très positive entre l'âge des ovocytes et le nombre d'embryons supposés triplö̈des (à 3 nucléoles) ; lorsque l'âge dépasse $20 \mathrm{~h}$, leur fréquence peut avoisiner 100 p. 100 ; l'utilisation de femelles mutées (diploïdes à 1 nucléole) leur permet de démontrer élégamment que les triploïdes résultent de rétentions accidentelles du second globule polaire.

Le facteur "vieillissement des ovules » pourrait par conséquent être en grande partie responsable des déviations du taux de diploïdie spontanée.

\section{F. La diploïdie induite}

L'utilisation de la gynogenèse spontanée pour l'obtention de lignées unisexuées est envisagée chez les espèces à forte fécondité ; cependant, sans élucider le mécanisme de la régulation diploïde, on ne peut appréhender le niveau de consanguinité des lignées obtenues.

II est donc apparu souhaitable d'élever sensiblement le taux de diploïdie spontanée en soumettant les œufs haploïdes à l'action d'agents antimitotiques classiques, connus pour provoquer la polyploïdie. Les œufs de vertébrés étant pondus en métaphase II, le traitement vise soit à induire la rétention du second globule polaire, ce qui revient à fusionner deux produits de méïose identiques aux post-réductions près ; soit plus rarement à inhiber la séparation des lots haploïdes fils issus de l'une des premières mitoses de segmentation.

\section{La rétention du second globule polaire par choc froid.}

Rostand fut le premier à suggérer (1934, 1936), qu'un choc froid de longue durée pouvait non seulement provoquer la gynogenèse, mais aussi la rétention du second globule polaire; de tels traitements ont en effet permis d'obtenir par la suite des quantités importantes d'individus triploïdes chez Triturus viridescens (Kankhauser et Griffiths, 1939 ; Griffiths, 1941), Triturus pyrrhogaster (Fankhauser et al., 1942) et Triturus alpestris (Fischberg, 1944). Fischberg (1947) fut le premier à obtenir par cette méthode des individus diploïdes gynogénétiques.

Toutes ces inductions de la triploïdie relatent l'application, juste après la fécondation in vivo, des chocs froids longs de plusieurs heures à des températures comprises entre 0 et $4{ }^{\circ} \mathrm{C}$, dont l'action provoque la formation de deux pronuclei femelles près du cortex de l'œuf (Beetschen, 1959). Le traitement conduit à de fortes mortalités embryonnaires et à des hétéroploïdies diverses, notamment à de nombreux haploïdes. Bien que certains travaux récents soient peu concluants, la reprise de ces expériences de choc froid par Kawahara (1978) (traitements de courtes durées) montre, que chez le xénope, le refroidissement devient un agent très compétitif pour provoquer la rétention du second globule polaire, lorsqu'il est appliqué avec une précision accrue (notamment après une fécondation in vitro).

C'est en tout cas la méthode la plus utilisée chez les poissons. En 1943, Makino et Ozima montrèrent que chez la carpe, le choc froid pouvait également entraîner la formation d'un pronucleus diploïde, mais sans produire d'individus triploïdes. Svärdson (1945) relate l'obtention d'un embryon triploïde hybride par la même méthode chez les salmonidés. Ce fut cependant Swarup (1958) chez l'épinoche, qui obtint le premier des rendements élevés de triploïdie. La diploïdisation des germes gynogénétiques est aujourd'hui obtenue aisément chez plusieurs 
espèces de poissons par l'emploi de températures comprises entre $0^{\circ} \mathrm{C}$ et $4{ }^{\circ} \mathrm{C}$ (Purdom, 1969 ; Nagy et al., 1978) ou comprises entre $-1{ }^{\circ} \mathrm{C}$ et $0^{\circ} \mathrm{C}$ (Purdom et Lincoln, 1974 ; Chourrout, 1980). Certaines études ne montrent pas une augmentation significative du taux de diploïdie spontanée (Cherfas, 1965 a).

Enfin, Thibault (1949) provoque la parthénogenèse et la rétention du second globule polaire, en apposant des glaçons sur les trompes de lapine ; les expériences du même type, visant à induire la triploïdie chez la souris (Beatty et Fischberg, 1949 ; Fischberg et Beatty 1950, 1952b) n'ont pas donné de résultats concluants.

2. La rétention du second globule polaire par choc chaud.

Un certain nombre d'études anciennes ont montré que des chocs chauds de courte durée à des températures voisines de $35^{\circ} \mathrm{C}$, appliqués durant la seconde division méiotique, provoquent la rétention du second globule polaire chez Triturus viridescens (Fankhauser et Watson, 1942), Rana pipiens (Briggs, 1947) et Xenopus laevis (Smith, 1958). Le succès et la reproductibilité de ces expériences explique l'utilisation courante des chocs chauds pour produire aujourd'hui des amphibiens triploïdes (Trottier et Armstrong, 1976 ; Ferrier et Jaylet, 1978 ; Cassidy et Blackler, 1980), ou diploïdes gynogénétiques ( Volpe et Dasgupta, 1962 ; Nace et al., 1970 ; Trottier et Armstrong 1976 ; Jaylet et Ferrier, 1978). Le taux de survie embryonnaire est souvent élevé et les survivants ont presque tous le caryotype désiré.

Sladececk et Lanzova (1959) ont montré que le traitement provoque une migration des chromosomes ovocytaires du cortex vers le centre de l'œuf, où se forme un pronucleus femelle diploïde. Cassidy et Blackler (1980) ont observé que chez le xénope, un traitement similaire, mais appliqué avant la fécondation pouvait être efficace, mais de manière plus aléatoire.

Les chocs chauds ont permis l'induction de la triploïdie chez les poissons (Swarup, 1958 ; Valenti, 1975 ; Chourrout, 1980) et plus récemment la diploïdisation de germes gynogénétiques (Chourrout, 1980).

Enfin, un pourcentage assez faible $(11$ p. 100) d'embryons triploïdes a été produit chez la souris par immersion des trompes de Fallope à une température voisine de $45^{\circ} \mathrm{C}$ (Beatty et Fischberg, 1949; Fischberg et Beatty, 1950, 1952 b).

3. La rétention du second globule polaire par choc de pression.

Rugh et Marsland en 1943, décrivant chez Rana pipiens les anomalies du développement consécutives à l'application de chocs de pression hydrostatique (10 000 psi durant $17 \mathrm{~min}$.) entre l'insémination et le $3^{\mathrm{e}}$ clivage, ont suggéré la possibilité d'induire la triploïdie à l'aide de traitements similaires, mais moins drastiques. Dasgupta (1962) a effectivement montré que le fuseau de méiose II disparaissait à la suite d'un traitement appliqué $5 \mathrm{~min}$. après la fécondation $(5000$ psi durant $6 \mathrm{~min}$.) ; ce phénomène provoque la triploïdisation dans 85 p. 100 des cas.

Ce type de méthode est aujourd'hui largement utilisé chez les amphibiens pour induire la triploïdie (Tompkins, 1978 ; Ferrier et Jaylet, 1978) et la gynogenèse diploïde (Tompkins, 1978 ; Jaylet et Ferrier, 1978 ; Gillespie et Armstrong, 1979) ; le choc doit être appliqué avant l'initiation de l'anaphase II, c'est-à-dire un peu plus tôt que le choc chaud; les efficacités de ces deux traitements sont du même ordre. 
Enfin, il a été mis au point récemment chez la truite arc-en-ciel, permettant d'obtenir des individus gynogénétiques et triploïdes (Chourrout, en préparation).

\section{La rétention du second globule polaire chez les mammifères.}

Nous avons vu que la plupart des traitements qui se sont révélés efficaces chez les amphibiens et chez les poissons n'ont guère donné de résultats chez les mammifères. La mise au point de la fécondation in vitro et de l'induction de la parthénogenèse in vitro a favorisé la reprise des tentatives de rétention du second globule polaire à l'aide d'autres agents :

a. La colchicine. - Le traitement des œufs par la colchicine lou par la colcémide), un inhibiteur du fuseau de division, a fait l'objet de nombreux travaux à partir des années 50 . Le résultat le plus probant est celui de Bomsel-Helmreich et Thibault (1962), qui ont obtenu le lapin 97 p. 100 d'œufs triploïdes pour un taux de fécondation in vitro de 65 p. 100. Une étude plus récente (MacGauchey et Chang, 1969) montre que l'injection de colchicine à une souris $3 \mathrm{~h} 30$ avant l'ovulation induit la triploïdie dans 6 cas sur 20 pour un taux de fécondation in vivo de 26 p. 100, mais aucun des œufs ne s'est clivé.

b. La cytochalasine $B$. - Cet agent inhibiteur de la cytodiérèse est le plus efficace chez la souris. La triploïdie a été induite aussi bien après une fécondation in vivo (Niemerko, 1975) qu'in vitro (Niemerko et Komar, 1976). Des embryons parthénogénétiques ont été obtenus par la même méthode par Balakier et Tarkowski (1976).

c. Le choc osmotique. - La triploïdie a été induite chez la souris (Opas, 1977) en soumettant les œufs fécondés à un choc osmotique brutal (eau distillée). Cette technique est pour le moment moins efficace que l'emploi de cytochalasine $B$.

\section{La suppression d'une des premières mitoses.}

Les études relatant le doublement du stock chromosomique par la suppression d'une des premières mitoses sont nettement moins nombreuses. Si la production d'embryons tétraploïdes est déjà ancienne, celle de diploïdes homozygotes n'a fait l'objet que de trois publications récentes.

a. La production de tétraploïdes par choc chaud. - Le premier succès fut enregistré chez la souris (Fischberg et Beatty, 1950 ; Beatty et Fischberg, 1952 a, b), après un réchauffement in situ des trompes de Fallope $\left(5 \mathrm{~min}\right.$. à $\left.45,5^{\circ} \mathrm{C}\right)$ au moment présumé de l'apparition du premier clivage : 7 embryons tétraploïdes ont été recensés sur un total de 60 recueillis trois jours et demi après la copulation. Fischberg, en 1958, après une fécondation in vitro chez Triturus vulgaris soumit 62 œufs triés à vue et venant de terminer leur premier clivage, à $35,6-36^{\circ} \mathrm{C}$ pendant 10-12 $\mathrm{min}$. et il recensa 16 survivants dont 15 tétraploïdes. Cette méthode a été reprise avec succès chez d'autres urodèles par Romanovski et Spicarova (1961) et Jaylet (1972), et chez les anoures par Kawamura et Nishioka (1960), Kawamura et al. (1963) et Nishioka (1971). Gaillard et Jaylet (1975) ont montré que les œufs doivent être chauffés peu après l'apparition du premier sillon de segmentation, moment où ils présentent deux noyaux interphasiques prémitotiques. II y a alors inhibition de la migration des asters vers les pôles opposés de chacun des deux noyaux, ce qui provoque la formation d'un fuseau courbe qui ne peut 
répartir les chromosomes en deux lots éloignés, d'où la formation de deux noyaux tétraploïdes.

Deux études préliminaires visant à produire le même phénomène (Thorgaard et al., 1981 ; Chourrout, 1982) ont donné quelques individus tétraploïdes chez la truite arc-en-ciel.

Les efforts pour provoquer de la même manière la diploïdisation de germes haploïdes gynogénétiques chez le triton Pleurodeles waltlii (Jaylet, 1972) n'ont pas été concluants.

b. L'utilisation de la pression hydrostatique. - Un traitement des œufs de xénope en métaphase de 1 re division $(6 \mathrm{~min}$. à 7000 psi) a permis d'obtenir 100 p. 100 d'embryons tétraplö̈des pour une survie de 50 p. 100 (Reindschmidt et al., 1979) ; les auteurs prétendent d'autre part avoir produit à l'aide de la même technique 3 individus gynogénétiques homozygotes ayant dépassé la métamorphose, mais sans préciser le nombre d'œufs traités : ils précisent que les géniteurs utilisés provenaient de souches déjà consanguines.

Streisinger et al. (1981) en combinant l'action de l'éther avec un choc de pression similaire sur les œufs du poisson Brachydanio rerio fécondés avec du sperme traité par les UV, ont obtenu 20 p. 100 d'embryons normaux dont 20 p. 100 ont atteint la maturité sexuelle ; l'éventualité d'une consanguinité élevée de la population utilisée n'est pas mentionnée. Récemment, un choc de pression à 7000 psi durant 3-4 min. et appliqué $5 \mathrm{~h} 50$ après la fécondation a permis d'obtenir 100 p. 100 d'individus tétraploïdes chez la truite arc-en-ciel (Chourrout, en préparation).

c. L'utilisation de la cytochalasine $B$. - Elle a permis la production d'embryons tétraploïdes chez la souris (Snow, 1973), à partir d'œufs traités in vitro pendant $12 \mathrm{~h}$ au stade 2 cellules. La répétition de ce type d'expériences sur des œufs haploïdes (après extraction d'un pronucleus) a conduit à des développements diploïdes (Markert et Peters 1977) ; les animaux utilisés appartenaient à des lignées consanguines.

\section{G. Conclusion générale}

\section{L'évolution des techniques d'induction.}

A partir des années soixante, la mise en pratique de traitements optimisés s'est effectuée chez les amphibiens, puis chez les poissons.

Aujourd'hui, la gynogenèse diploïde est induite chez les amphibiens le plus souvent à l'aide de la combinaison d'un traitement du sperme par les rayons UV (parce que celui-ci est très facilement réalisable en laboratoire), et d'un choc chaud ou hyperbare des œufs (déclarés plus efficaces que les chocs froids).

Chez les poissons, la mode est à la combinaison rayons ionisants-chocs froids ce qui traduit un retard, mais aussi le fait que les chocs froids se sont révélés particulièrement efficaces dans plusieurs cas : la raison est ici peut-être liée à la pratique systématique de l'insémination in vitro, qui assure en théorie un synchronisme des développements embryonnaires, alors que le test des chocs froids a été effectué chez les amphibiens dans le cadre de la triploïdie induite, à 
une époque où on ne pratiquait que l'insémination in vivo. On note toutefois chez les poissons une évolution vers la combinaison utilisée chez les amphibiens. II semble par ailleurs dans les deux cas que le choc hyperbare soit le meilleur agent d'inhibition de la première mitose de segmentation.

Dans le cas des mammifères, l'induction de la gynogenèse a été abandonnée à la suite d'un certain nombre d'insuccès, et a fait place à l'induction de la parthénogenèse ; la recherche d'un traitement de diploïdisation a été visiblement effectuée indépendamment des travaux sur les œufs d'amphibiens.

II ne semble pas que la découverte des effets spécifiques de chacun des agents au niveau cellulaire et moléculaire (sur cellules en culture et sur œufs d'invertébrés) ait provoqué une réelle discussion sur le choix du traitement optimal d'induction de la gynogenèse. Une première approche simple, visant à vérifier la conformité des produits obtenus (caryotypes pour l'absence de résidus de chromatine mâle et l'intégrité du stock diploïde ; méthodes biochimiques pour la nonexpression d'allèles paternels) est le plus souvent évitée par l'emploi de méthodes indirectes et peu rigoureuses (dénombrement des nucléoles, mesure de la taille des noyaux d'érythrocytes, unicité du marqueur de gynogenèse).

\section{L'apport de la gynogenèse induite chez les amphibiens.}

Grâce au grand nombre de travaux réalisés, il est aisé d'obtenir chez les amphibiens des individus gynogénétiques. Les rendements obtenus au stade de la métamorphose sont souvent faibles, ceci pouvant être en grande partie attribué à la mauvaise réussite de l'insémination in vitro. II semble aujourd'hui que la vague importante des travaux de mise au point de la gynogenèse induite ne soit guère justifiée par les profits réels qui en ont été tirés :

a. Les espérances de découverte de mutations récessives ont été déçues ; la gynogenèse se serait de ce point de vue peut-être révélée plus efficace, si elle avait été appliquée après des opérations de mutagenèse induite.

b. L'obtention de clones pour l'expérimentation a été le plus souvent réalisée par greffe nucléaire. Cette technique présentait un gros avantage : malgré ses très faibles rendements et la difficulté de sa mise au point, elle fournissait d'emblée des clones de quelques individus hétérozygotes, alors que la gynogenèse aboutissait le plus souvent après plusieurs générations à des individus homozygotes en faible nombre, fragiles et difficiles à croiser. D'autre part, la greffe nucléaire possédait d'autres débouchés, notamment l'étude de la différenciation des noyaux somatiques.

c. En ce qui concerne l'identification du mode de déterminisme du sexe chez une espèce donnée, elle a été devancée par le croisement de mâles obtenus par inversion sexuelle, qui fournit beaucoup plus d'individus sexables. D'autre part, la sex-ratio obtenue par gynogenèse s'écarte parfois des prévisions : chez Rana pipiens, espèce démontrée de type femelle homogamétique par inversion sexuelle, Volpe et Dasgupta (1962) obtiennent par gynogenèse 6 mâles et 11 femelles ; à la suite de la même observation, Richards et Nace (1978) ont démontré par recroisement le génotype femelle de ces néomâles ; cet écart du phénotype peut-il être mis au compte d'une sensibilité extrême des génomes consanguins aux conditions du milieu ? il démontre en tout cas, que l'utilisation de la gynogenèse pour 
identifier le type de déterminisme du sexe suivi par une espèce donnée est discutable.

d. Dans le domaine de l'étude de la différenciation sexuelle chez les urodèles, la gynogenèse a connu cependant une application intéressante quoique très ponctuelle : en effet, l'obtention de populations monosexes femelles chez une espèce mâle homogamétique requiert la production préalable de femelles $W W$. De ce point de vue, le croisement de mâles obtenus par inversion sexuelle fournit moins d'une fois sur deux une descendance comportant de tels génotypes, et ceux-ci ne représentent alors qu'un tiers des femelles obtenues, ce qui impose un testage laborieux sur descendance.

La gynogenèse, quant à elle, fournit d'emblée une descendance bisexuée et peu nombreuse, mais où toutes $\left(^{*}\right)$ les femelles sont de génotype WW.

e. Enfin, il n'y a pas eu de débouchés importants de la gynogenèse dans le domaine de la cartographie génétique.

\section{L'apport de la gynogenèse induite chez les mammifères.}

Les techniques d'induction de la gynogenèse, concernant principalement la souris, ont eu des rendements très faibles, voire nuls, et n'ont pas fourni d'adultes, mis à part le cas des expériences au cours desquelles le pronucleus mâle était prélevé à l'aide d'une micropipette : il convient à nouveau de préciser que les géniteurs utilisés appartenaient à des lignées consanguines. De ce point de vue, et à défaut de pcuvoir concurrencer le croisement frère-soeur, ce type de gynogenèse peut être envisagé comme un moyen ultime de fixer l'homozygotie dans le cas des lignées commerciales de rongeurs dont la pureté est parfois mise en doute.

4. Les perspectives de la gynogenèse induite chez les poissons.

Dans le cas des poissons d'intérêt économique, la gynogenèse induite semble avoir une portée plus générale. Les rendements obtenus sont satisfaisants, grâce à l'excellente survie après insémination in vitro, et la fertilité des animaux est par ailleurs bien plus grande.

a. Son utilisation dans le but de découvrir des mutants n'est pas envisagée et le clonage pour l'expérimentation est ici une préoccupation secondaire.

b. On recherche surtout la production de lignées consanguines à recroiser, et à cet égard, ni le croisement frère-sœur (voie trop lente), ni l'autofécondation d'individus hermaphrodites (obtenus difficilement par traitement hormonal) n'opposent de concurrence réelle.

Néanmoins, la valeur commerciale du schéma complet reste à tester ; la production de lignées grâce à la gynogenèse par rétention du second globule polaire nécessite plusieurs générations avant le croisement terminal ; sa réussite dépend entre autres d'un traitement d'induction optimisé et d'un contrôle rigoureux des produits.

Quant aux estimations de la vitesse d'élévation de la consanguinité, elles peuvent révéler des surprises, voire des déceptions : Purdom et al. (1976) sur la plie,

$\left(^{*}\right)$ En réalité, elle produit une minorité très faible de femelles ZW, provenant d'ovogonies tétraploides ZZWW, ou d'une recombinaison des facteurs sexuels. 
Cherfas et Truwelier (1978) sur la carpe $\left({ }^{*}\right)$, et Guyomard (en préparation) sur la truite arc-en-ciel $\left(^{*}\right)$ ont mesuré pour certains systèmes biochimiques une hétérozygotie résiduelle étonnament élevée. La mise au point de la gynogenèse par endomitose peut de ce point de vue se révéler très prometteuse (si son rendement n'est pas trop faible) dans la mesure où elle garantit la fixation immédiate de tous les loci.

c. La gynogenèse peut dès aujourd'hui, au moins chez les salmonidés, être considérée comme une voie très compétitive pour la production de populations monosexes femelles (Chevassus et al., en préparation). Elle fournit des individus de génotype femelle mais fragiles et inutilisables en élevage ; cependant, après inversion hormonale, tous les mâles gynogénétiques obtenus donnent par croisement avec des femelles standard des populations monosexes femelles non consanguines.

d. Enfin, les techniques de rétention du second globule polaire, appliquées à la suite d'inséminations par du sperme normal, fournissent des individus triploides dont la stérilité éventuelle est particulièrement prisée dans diverses formes d'aquaculture (contrôle de la reproduction, réduction des mortalités survenant lors de la maturation sexuelle, augmentation de l'indice de carcasse).

Reçu en octobre 1981.

Accepté en février 1982.

\section{Références}

AUSTIN C. R., BRADEN A. W. H., 1954. Anomalies in rat, mouse and rabbit eggs. Aust. J. biol. Sci., 7, 537-542.

BALAKIER H., TARKOWSKI A. K., 1976. Diploid parthenogenetic mouse embryos produced by heat-shock and cytochalasin B. J. Embryol. exp. Morph., 35, 25-39.

BATAILLON E., 1911. L'embryogenèse provoquée chez l'œuf vierge d'amphibiens par inoculation de sang ou de sperme de mammifère. Parthénogenèse traumatique et imprégnation sans amphimixie. C. R. Acad. Sci., 152, 1271-1273.

BEATTY R. A., FISCHBERG M., 1949. Spontaneous and induced triploidy in pre-implantation mouse eggs. Nature, London, 163, 807-808.

BEATTY R. A., FISCHBERG M., 1951. Heteroploidy in mammals. I. Spontaneous heteroploidy in pre-implantation mouse eggs. J. Genet., 50, 345-359.

BEATTY R. A., FISCHBERG M., 1952. Heteroploidy in mammals. III. Induction of tetraploidy in pre-implantation mouse eggs. J. Genet., 50, 471-479.

BEETSCHEN J. C., 1959. Modifications des structures nucléaires et cytologiques de l'œuf fécondé et insegmenté soumis à une réfrigération prolongée chez le Triton Pleurodeles waltiii Michah. C. R. Acad. Sci. Paris, 249, 173-175.

BLACKLER A. W., CASSIDY D. M., 1980. Developmental and ploidy characteristics of " senescent " unfertilized eggs of Xenopus laevis. J. exp. Zool, 213, 105-115.

BOMSEL-HELMREICH O., THIBAULT C., 1962. Fécondation in vitro en présence de colchicine et polyploidie expériementale chez le lapin. Ann. Biol. anim. Bioch. Biophys., 2, 13-16.

BRADEN A. W. H., 1957. Varaiations between strains in the incidence of various abnormalities of egg maturation and fertilization in the mouse. J. Genet., 55, 476-486. fixée $"$.

(*) Encore que dans le cas d'espèces supposées tétraploïdes, il puisse s'agir d'une « hétérozygotie 
BRIGGS R., 1947. The experimental production and development of triploid frog embryos. J. exp. Zool., 106, 237-266.

BRIGGS R., GREEN E. U., KING T. J., 1951. An investigation of the capacity for cleavage and differentiation in Rana pipiens eggs lacking functional chromosomes. J. exp. Zool., 116, 455-498.

BUSS K. W., WRIGHT J. E. Jr., 1956. The results of species hybridization within the family Salmonidae. Progr. Fish. Cult., 18, 149-158.

CASSIDY D. M., BLACKLER A. W., 1980. Induction of triploidy in Xenopus laevis by heat shocking of the egg before and after fertilization. J. exp. Zool., 211, 379-385.

CHANG M. C., HUNT M. C., ROMANOFF E. B., 1957. Effects of radiocobalt irradiation of rabbit spermatozoa in vitro on fertilization and early development. Anat. Rec., 129, 211-224.

CHERFAS N. B., 1965 a. Natural triploidy in females of the unisexual form of silver carp (goldfish) (Carassius auratus gibelio Bloch). Genetika, 5, 16-24.

CHERFAS N. B., $1965 \mathrm{~b}$. Investigation of radiation induced diploid gynogenesis in the carp (Cyprinus carpio L.). I. Experiments on obtaining the diploid gynogenetic progeny in mass quantities (en russe). Genetika, 11, 78-86.

CHERFAS N. B., TRUWELLER K. A., 1978. Investigation of radiation induced diploid gynogenesis in the carp (Cyprinus carpio L.). III. Gynogenetic offspring analysis by biochemical markers (en russe). Genetika, 14, 599-604.

CHOURROUT D., 1980. Thermal induction of diploid gynogenesis and triploidy in the rainbow trout (Salmo gairdneri Richardson). Reprod. Nutr. Dévelop., 20, 727-733.

CHOURROUT D., 1982. Tetraploidy induced by heat shocks in the rainbow trout (Salmo gairdneri R.). Reprod. Nutr. Dévelop., 22, 569-574.

CHOURROUT D., CHEVASSUS B., HERIOUX F., 1980. Analysis of an Hertwig effect in the rainbow trout (Salmo gairdneri Richardson) after fertilization with gamma-irradiated sperm. Reprod. Nutr. Dévelop., 20, 719-726.

CIMINO M. C., 1972. Meiosis in triploid all-female fish (Poeciliopsis, Poecilidae). Science, 175, 14841486.

DALCO A., 1931, Contribution à l'analyse des fonctions nucléaires dans l'ontogenèse de la grenouille. I. Etude cytologique des œufs fécondés par du sperme trypaflaviné. Arch. Biol. Paris, 41, 143-220.

DALCO A., SIMON S., 1931. Contribution à l'analyse des fonctions nucléaires dans l'ontogenèse de la grenouille. III. Etude statistique et cytologique des effets de l'irradiation d'un des gamètes sur la gastrulation chez Rana fusca. Arch. Biol. Paris, 42, 107-165.

DASGUPTA S., 1962. Induction of triploidy by hydrostatic pressure in the leopard frog, Rana pipiens. J. exp. Zool., 151, 105-116.

DREBINGER K., 1951. Kerngifte und Lichtstrahlung. Eine Studie an Frosch-spermien zur Wirkungsanalyse der Kerngifte. Roux Arch. Entw. Mech. Organ., 145, 174-204.

EDWARDS R. G., 1956 a. The experimental induction of gynogenesis in the mouse. I. Irradiation of the sperm by X-rays. Proc. roy. Soc., 146, 469-487.

EDWARDS R. G. $1956 \mathrm{~b}$. The experimental induction of gynogenesis in the mouse. II. Ultraviolet irradiation of the sperm. Proc. roy. Soc., 146, 486-504.

EDWARDS R. G., 1958. The experimental induction of gynogenesis in the mouse. III. Treatment of sperm with trypaflavine, toluidine blue, nitrogen mustard. Proc. roy Soc., 149, 117-129.

FANKHAUSER G., CROTTA R., PERROT M., 1942. Spontaneous and cold-induced triploidy in the japanese newt, Triturus pyrrhogaster. J. exp. Zool., 89, 167-181.

FANKHAUSER G., GRIFFITHS R. B., 1939. Induction of triploidy and haploidy in the newt Triturus viridescens by cold treatment of unsegmented eggs. Proc. nat. Acad. Sci. (Wash.), 25, 233-238.

FANKHAUSER G., HUMPHREY R. R., 1959. The origin of spontaneous heteroploids in the progeny of diploid, triploid and tetraploid axolotl females. J. exp. Zool., 142, 379-422.

FANKHAUSER G., WATSON R. C., 1942. Heat-induced triploidy in the newt Triturus viridescens. Proc. nat. Acad. Sci. (Wash.), 28, 436-440.

FARINA E., 1976. Diploid gynogenetics in Discoglossus pictus. Acta embryol. exp., 3, 291-297. 
FERRIER V., 1966. Gynogenèse diploïde et polyplö̈de réalisée expérimentalement chez le Triton Pleurodeles waltlii. C. R. Soc. Biol. (Paris), 160, 1526-1531.

FERRIER V., JAYLET A., 1978. Induction of triploidy in the newt Pleurodeles waltlii by heat shock and hydrostatic pressure. Interpretation of the different types of ploidy using a chromosomal marker. Chromosoma, 69, 47-63.

FISCHBERG M., 1944. Veranderungen der Chromosomenzahl bei Triton alpestris nach Kaltebehandlung der Eier. Rev. suisse Zool., 51, 430-436.

FISCHBERG M., 1947. Experimentelle Auslosung von haploider zur diploider Parthenogenese bei den Urodelen Triton palmatus und Triton alpestris. Arch. Klaus-Stift. Vererbforsch., 22, 331-336.

FISCHBERG M., 1958. Experimental tetraploidy in newts. J. Embryol. exp. Morph., 6, 393-402.

FISCHBERG M., BEATTY R. A., 1950. Experimentelle Herstellung von polyploiden Mausblastulae. Arch. Klaus-Stift. Vererbforsch., 25, 54-55.

FISCHBERG M., BEATTY R. A., 1952 a. Heteroploidy in mouse embryos due to crossing inbred strains. Evolution, 6, 216-324.

FISCHBERG M., BEATTY R. A., 1952 b. Heteroploidy in mammals. II. Induction of triploidy in pre- implantation mouse eggs. J. Genet., 50, 455-470.

FRASER L. R., 1971. Physico-chemical properties of an agent that induces parthenogenesis in Rana pipiens eggs. J. exp. Zool., 177, 153-172.

GAILLARD G., JAYLET A., 1975. Mécanisme cytologique de la tétraplö̈die expérimentale chez le Triton Pleurodeles waltiii. Chromosoma, 51, 125-133.

GILLESPIE L. L., ARMSTRONG J. B., 1979, Induction of triploid and gynogenetic diploid axolotls (Ambystoma mexicanum) by hydrostatic pressure. J. exp. Zool., 210, 117-122.

GOLUBOVSKAYA I. N., 1979. Genetic control of meiosis. Int. Rev. Cytol., 58, 247-290.

GRIFFITHS R. B., 1941. Triploidy and haploidy in the newt Triturus viridescens, induced by refrigeration of fertilized eggs. Genetics, 26, 69-88.

HERTWIG G., 1924. Trypaflavin als Radiumersatz zur Gewinnung haploid kerniger Froschlarven. Verh. Anat. Ges. (Jena), 58, 223-227.

HERTWIG O., 1911. Die Radiumkrankheit tierischer Keimzellen. Arch. Mikr. Anat., 7, 1-97.

HUBBS C. L., HUBBS L. C., 1932. Apparent parthenogenesis in nature, in a form of fish of hybrid origin. Science, 76, 628-630.

HUBBS C. L., 1961. Isolating mechanisms in the speciation of fishes, 5-23. In W. Franck BLAIR, Vertebrate speciation. Univ. Texas Press, Austin (USA).

HUMPHREY R. R., FANKHAUSER G., 1957. The origin of spontaneous and experimental haploids in the mexican axolotl (Siredon- or Ambystoma mexicanum). J. exp. Zool., 134, 427-447.

IWAMATSU T., OHTA T., 1974. Cleavage initiating activities in sperm fractions injected into the egg of the medaka, Oryzias latipes. J. exp. Zool, 187, 3-12.

JAYLET A., 1972. Tétraploïdie expérimentale chez le triton Pleurodeles waltlii Michah, Chromosoma, 38, 173-184.

JAYLET A., 1972. Recherches sur diverses modifications expérimentales de l'assortiment chromosomique chez le triton Pleurodeles waltii. Remaniement du caryotype, tétraploïdie, gynogenèse. Th. Doct. Sc. nat., $\mathrm{N}^{\circ}$ 466, Univ. Paul Sabatier, Toulouse.

JAYLET A., FERRIER V., 1978. Experimental gynogenesis in the newt species Pleurodeles waltiii and $P$. poireti. Chromosoma, 69, 65-80.

KALLMAN K. D., 1962. Population genetics in the gynogenetic teleost Mollienesia formosa (Girard). Evolution, 16, 497-504.

KAWAHARA H., 1978. Production of triploid and gynogenetic diploid Xenopus by cold treatment. Develop., Growth Differ., 20, 227-236.

KAWAMURA T., 1942. III. Triploid frogs developed from fertilized eggs. Proc. imp. Acad. Tokyo, 17, 523-526.

KAWAMURA T., NISHIOKA M., 1960. Amphidiploids produced by artificial means. J. Sci. Hiroshima Univ., Ser. B, Div. 1, 18, 195-201.

KAWAMURA T., NISHIOKA M., MYOREI Y., 1963. Reproductive capacity of autotetraploid males in brown frogs. J. Sci. Hiroshima Univ., Ser. B, Div. 1, 21, 1-10.

KOBAYASI H., 1976. A cytological study on the maturation division in the oogenic process of the triploid Ginbuna (Carassius auratus langsdorfii). Jap. J. Ichthyol., 22, 234-240. 
LASHER R., RUGH R., 1962. The " Hertwig " effect in teleost development. Biol. Bull., 123, 582588.

LEMOINE H. L. Jr., SMITH L. T., 1980. Polyploidy induced in brook trout by cold shock. Trans. amer. Fish. Soc., 109, 626-631.

LIEDER U., 1959. Uber die Eientwicklung bei mannchenlosen Stammen der Silverkarausche Carassius auratus gibelio (Bloch) (Vertebrata-Pisces). Biol. Zentralbl., 78, 284-291.

LINCOLN R. F., AULSTAD D., GRAMMELTVEDT A., 1974. Attempted triploid induction in atlantic salmon (Salmo salar) using cold shocks. Aquaculture, 4, 287-297.

MacGAUCHEY R. W., CHANG M. C., 1969. Inhibition of fertilization and production of heteroploidy in eggs of mice treated with colchicine. J. exp. Zool., 171, 465-480.

MAKINO S., OZIMA Y., 1943. Formation of the diploid egg nucleus, due to the suppression of the secund maturation division induced by refrigeration of fertilized eggs of the carp, Cyprinus carpio. Cytologia, Tokyo, 13, 55-60.

MARKERT C. L., PETERS R. M., 1977. Homozygous mouse embryos produced by microsurgery. J. exp. Zool., 201, 295-302.

MAUDLIN I., FRASER L. P., 1977. The effect of PMSG dose on incidence of chromosome anomalies in mouse embryos fertilised « in vitro ". J. Reprod. Fert., 50, 273-280.

MODLINSKI J. A., 1975. Haploid mouse embryos obtained by microsurgical removal of one pronucleus. J. Embryol. exp. Morph., 33, 897-905.

NACE G. W., RICHARDS C. M., ASHER J. H., 1970. Parthenogenesis and genetic variability. I. Linkage and inbreeding estimations in the frog Rana pipiens. Genetics, 66, 349-368.

NAGY A., RAJKI K., HORVATH L., CSANYI V., 1978. Investigation on carp, Cyprinus carpio L. gynogenesis. J. Fish Biol., 13, 215-224.

NIEMERKO A., 1975. Induction of triploidy in the mouse by cytochalasin B. J. Embyrol. exp. Morph., 34, 279-289.

NIEMERKO A., KOMAR A., 1976. Cytochalasin B-induced triploidy in mouse oocytes fertilized in vitro. J. Reprod. Fert., 48, 279-284.

NISHIOKA M., 1971. Abnormal combinations of the nucleus and the cytoplasm and their effects in amphibians. Brookhaven Symp. Cell Biol., 22, 189-203.

OPAS J., 1977. Effects of extremely low osmolarity on fertilized mouse eggs. J. Embryol. exp. Morph., 37, 65-77.

OPPERMANN K., 1913. Die Entwicklung von Forelleneiern nach Befruchtung mit radiumbestrahlten Samenfaden. Arch. Mikr. Anat., 83, 141-189.

PINCUS G., 1939. The comparative behaviour of mammalian eggs in vivo and in vitro. IV. The development of fertilized and artificially activated rabbit eggs. J. exp. Zool., 82, 85-130.

PINCUS G., ENZMANN E. V., 1936. The comparative behaviour of mammalian eggs in vivo and in vitro. II. The activation of tubal egg of the rabbit. J. exp. Zool., 73, 195-208.

POGANY G. C., 1976. Analysis of the Hertwig effect induced by the irradiation of Rana pipiens sperm with ultraviolet. Develop. Growth Differ., 18, 339-348.

PURDOM C. E., 1969. Radiation-induced gynogenesis and androgenesis in fish. Heredity, 24, 431-444.

PURDOM C. E., LINCOLN R. F., 1974. Gynogenesis in hybrids within the Pleuronectidae. The early life story of fish. Springer-Verlag, Berlin, Heidelberg, New York.

PURDOM C. E., THOMPSON D., DANDO P. R., 1976. Genetic analysis of enzyme polymorphisms in plaice (Pleuronectes platessa). Heredity, 37, 193-206.

RASCH R. M., DARNELL E. M., KALLMAN K. D., ABRAMOFF P., 1965. Cytophotometric evidence for triploidy in hybrids of the gynogenetic fish Poecilia formosa. J. exp. Zool., 160, 155-170.

REINDSCHMIDT D. C., SIMON S. J., VOLPE E. P., TOMPKINS R., 1979. Production of tetraploid and homozygous diploid amphibians by suppression of first cleavage. J. exp. Zool., 210, 137-143.

RICHARDS C. M., NACE G. W., 1977. The occurrence of diploid ova in Rana pipiens. J. Heredity, 68, 307-312.

RICHARDS C. M., NACE G. W., 1978. Gynogenetic and hormonal sex-reversal used in tests of the XX-XY hypothesis of sex-determination in Rana pipiens. Growth, 42, 319-331. 
ROMASHOV D. D., BELYAEVA V. N., GOLOVINSKAIA K. A., PROKOVEVA-BELGOVSKAYA A. A., 1961. Radiation disease in fish (en russe). Radiatsionnaya Genetika, Acad. Sci. USSR, Moscow, 247-266.

ROMASHOV D. D., NIKOLYUKIN N. I., BELYAEVA V. N., TIMOFEEVA N. A., 1963. Possibilities of producing diploid radiation-induced gynogenesis in sturgeons. Radiobiologiya, 3 , 104-109 (en russe). Traduit en anglais dans Radiobiology, 3. 145-154.

ROMANOVSKY A., SPICAROVA N., 1961. The production of tetraploid newts by Fischberg's method. Folia biol. (Prague), 7, 395-399.

ROSTAND J., 1934. Gynogenèse du crapaud par refroidissement de l'œuf. C. R. Soc. Biol. (Paris), 115, 1680-1681.

ROSTAND J., 1936. Gynogenèse par refroidissement des œufs chez Hyla arborea. C. R. Soc. Biol. (Paris), 122, 1012-1013.

RUGH R., 1939. Developmental effects resulting from exposure to X-rays. I. Effect on the embryo of irradiation of frog sperm. Proc. amer. Phil. Soc., 83, 447-471.

RUGH R., EXNER F., 1940. Developmental effects resulting from exposure to X-rays. II. Devevelopment of leopard frog eggs activated by bull-frog sperm. Proc. amer. Phil. Soc., 83, 607-619.

RUGH R., MARSLAND D. A., 1943. The effect of hydrostatic pressure upon the early development of the frog's eggs (Rana pipiens). I. Macroscopic observations. Proc. amer. Phil. Soc., 86, 459-466.

SCHULTZ R. J., 1967. Gynogenesis and triploidy in the viviparous fish Poeciliopsis. Science, 157. 1564-1567.

SCHULTZ R. J., KALLMAN K. D., 1968. Triploid hybrids between the all-female teleost Poecilia formosa and Poecilia sphenops. Nature, 219, 280-282.

SIMON G., 1972. Effets de l'irradiation des spermatozoïdes sur la fécondation de l'ceuf chez le lapin (Oryctolagus cuniculus). Th. $3^{\mathrm{e}}$ cycle, Univ. Paris VI.

SLADECEK F., LANZOVA J., 1959. Cytological mechanism of the production of heteroploids by cold or heat shocks in the axolotis. Folia biol., 5, 379-393.

SMITH S., 1958. Induction of triploidy in the south african clawed frog Xenopus laevis (Daudin). Nature, 181, 290.

SNOW M. H. L., 1973. Tetraploid mouse embryos produced by cytochalasin B during cleavage. Nature, 244, 513-515.

STANLEY J. G., 1976. Production of hybrid, androgenetic and gynogenetic grass carp and carp. Trans. amer. Fish Soc., 105, 10-16.

STANLEY J. G., JONES J. B., 1976. Morphology of androgenetic and gynogenetic grass carp Ctenopharyngodon idella (Valenciennes). J. Fish Biol., 9, 523-528.

STREISINGER G., WALKER C., DOWER N., KNAUBER D., SINGER F., 1981. Production of clones of homozygous diploid zebrafish (Brachydanio rerio). Nature, 291, 293-296.

SVÄRDSON G., 1945. Chromosome studies on salmonidae. Rep. Swedish State Inst. Freshwater Fishery Res., Drottningholm, No 23.

SWARUP H., 1958. Productin of triploidy in Gasterosteus aculeatus. J. Genetics, 56, 129-142.

THIBAULT C., 1949. L'œuf des mammifères. Son développement parthénogénétique. Ann. Sci. nat. (Zool.), 11, 136-219.

THIBAULT C., ORTAVANT R., 1949. Parthénogenèse expérimentale chez la brebis. C. R. Acad. Sci. Paris, 228, 510-511.

THORGAARD G. H., JAZWIN M. E., STIER A. R., 1981. Polyploidy induced by heat shock in rainbow trout. Trans, amer. Fish. Soc., 110, 546-550.

TING H., 1951. Diploid androgenetic and gynogenetic haploid development in anuran hybridization. J. exp. Zool., 116, 21-58.

TOMPKINS R., 1978. Triploid and gynogenetic diploid Xenopus laevis. J. exp. Zool., 203, 251-256.

TROTTIER T. M., ARMSTRONG J. B., 1976. Diploid gynogenesis in the mexican axolotl. Genetics, 83, 783-792.

TSOY P. M., 1969. Action de la nitrosolméthylurée et du diméthylsulfate sur le sperme de truite arc-en-ciel et de corégone (en russe). Dok. Akad. Nauk. USSR, 189, 411-414.

UZZELL T., 1964. Relations of the diploid and triploid species of the Ambystoma jeffersionium complex (Amphibia, caudata). Copeia, 2, 257-300. 
UZZELL T., 1970. Meiotic mechanisms of naturally occuring unisexual vertebrates. Amer. Natur., 104, 443-445.

VALENTI R. F., 1975. Induced polyploidy in Tilapia aurea (Steindachner) by means of temperature shock treatment. J. Fish Biol., 7, 519-528.

VOLPE E. P., DASGUPTA S., 1962. Gynogenetic diploids of mutant leopard frogs. J. exp. Zool., 151, 287-301.

WILLIAMS C. V., LONG S. E., 1980. The effect of superovulation on the chromosome complement of early sheep embryos. 4th eur. Coll. Cytogenet. domest. Anim., 168-171.

WITSCHI E., LAGUENS R., 1963. Chromosomal abberations in embryos from overripe eggs. Dev. Biol., 7, 605-616. 